\title{
Titan under a red giant sun: A new kind of "habitable" moon
}

\author{
Ralph D. Lorenz, Jonathan I. Lunine \\ Lunar and Planetary Laboratory, Department of Planetary Sciences, University of Arizona, Tucson, AZ \\ 85721-0092, USA \\ Christopher P. McKay \\ NASA Ames Research Center, Moffett Field, CA 94035, USA
}

\begin{abstract}
We explore the response of Titan's surface and massive atmosphere to the change in solar spectrum and intensity as the sun evolves into a red giant. Titan's surface temperature is insensitive to insolation increases as the haze-laden atmosphere "puffs up" and blocks more sunlight. However, we find a window of several hundred Myr exists, roughly 6 Gyr from now, when liquid water-ammonia can form oceans on the surface and react with the abundant organic compounds there. The window opens due to a drop in haze production as the ultraviolet flux from the reddening sun plummets. The duration of such a window exceeds the time necessary for life to have begun on Earth. Similar environments, with $\sim 200 \mathrm{~K}$ water-ammonia oceans warmed by methane greenhouses under red stars, are an alternative to the $\sim 300 \mathrm{~K}$ water- $\mathrm{CO}_{2}$ environments considered the classic "habitable" planet.
\end{abstract}

\section{Introduction}

In about 7 billion years, the sun will complete its main sequence and become a red giant, incinerating the Earth [Sackmann et al. 1993]. However, other bodies may become more hospitable as the volatile-rich outer solar system is thawed out.

Recent work on planetary habitability [Williams et al.,1996] has focussed on the ability of $\mathrm{CO}_{2} / \mathrm{H}_{2} \mathrm{O}$ greenhouse atmospheres to maintain planetary surface temperatures above the water freezing point, the rationale being that terrestrial life relies for its formation and continuation on the presence of liquid water to mediate chemical reactions. It is well known that water-ammonia mixtures remain liquid down to $176 \mathrm{~K}$ [e.g. Croft et al. 1988]. While water-ammonia would make Earth's oceans and atmosphere toxic to its present biota, it may be a viable solvent for prebiotic and protobiotic chemistry elsewhere - like water itself, ammonia solutions are stable as liquid over a wide temperature range and have a high latent heat, stabilizing the environment [Shapiro and Feinberg, 1995]. The strongly-polar and hydrogen-bonding nature of water-ammonia is also favorable for protein-folding properties and for solvating the charged molecules common in biochemistry.

Another vital ingredient in the primordial soup is organics: it is well-known that complex molecules can be built up

Copyright 1997 by the American Geophysical Union.

Paper number 97GL52843.

0094-8534/97/97GL-52843\$05.00 by methane photochemistry in reducing atmospheres such as those of the outer planets. Although methane and ammonia have been considered as solutions to the faint early sun problem [Sagan and Mullen, 1977; Kiehl and Dickinson, 1987] such an atmosphere could not last long under terrestrial conditions - intense solar UV (100 times the flux at Titan for a given epoch, due to its proximity to the sun) would destroy ammonia directly [Kuhn and Atreya, 1977; but see also Sagan and Chyba, 1997].

The combination of a cool $(\sim 200 \mathrm{~K})$ water-ammonia surface, with a methane greenhouse, allows an aqueous liquid surface at much lower solar fluxes. At these low temperatures, the vapor pressures above the water-ammonia liquid are tiny [Haudenschild, 1970] $\left(10^{-4}\right.$ and $10^{-7}$ bar for ammonia and water respectively at $176 \mathrm{~K}$ ) - so the oxygen content of the atmosphere stays low and the low solar flux provides few UV photons for photolysis. Thus, such an environment could be relatively long-lived. Lower temperatures also slow the rate of Jeans escape. Titan provides us with a natural laboratory to investigate such an environment.

\section{Radiative Models}

In radiative terms, Titan's atmosphere may be considered as two distinct layers [Samuelson, 1983; McKay et al., 1989,1990] - a haze-laden stratosphere which absorbs sunlight, and a thick troposphere of greenhouse gases, opaque to thermal radiation. At present, its surface temperature is $94 \mathrm{~K}$, due to a $21 \mathrm{~K}$ greenhouse effect offset in part by a $9 \mathrm{~K}$ antigreenhouse - the haze absorbs about $60 \%$ of the $(3.7$ $\mathrm{Wm}^{-2}$ ) insolation. Experimenting with a detailed radiativeconvective (r-c) model [McKay et al., 1989] we find that Titan is only modestly sensitive to increased insolation. This is initially surprising given that earlier work had noted Titan's significant sensitivity to heating at the surface, caused by an increase in IR optical depth with temperature [McKay et al., 1993]. This sensitivity assumes a reservoir of volatiles (a hydrocarbon/nitrogen ocean) on the surface - increasing the surface temperature increases the vapor pressure of these greenhouse gases.

In the absence of such a feedback (if, for example, there is no ocean of methane on the surface) the sensitivity to heating is much less. Further, heating optically from above rather than simply injecting heat at the surface introduces an important negative feedback - the column haze opacity increases. This occurs because, while the haze is produced at a roughly fixed pressure level (about $10^{-4}$ bar [McKay et al., 1989], where UV is absorbed), the altitude this corresponds to increases as the atmospheric temperature (and hence scale 
height) rises. If production and removal (coagulation, condensation) processes remain constant, there are proportionally more haze particles in the column (as the scale height is larger), and so the opacity increases. Our experiments with the $\mathrm{r}-\mathrm{c}$ model indicate that although the altitude at which methane becomes saturated (and presumably removes haze by rainout) does depend on insolation and methane abundance, the effect is small; e.g. we varied the haze production rate over 5 orders of magnitude, and the optical depth changed by four orders of magnitude, yet the difference between haze optical depths for cases with and without methane rainout was only $20 \%$ - this is because only the bottom of the haze layer is affected by the rainout altitude.

The r-c model is not well-suited to study Titan with very high solar fluxes, as the absorption coefficients, haze microphysics etc. are optimised for the present cold conditions. We therefore use a simpler model to explore the response of Titan to extreme solar evolution. Our model has a grey IR-opaque, sunlight-transparent troposphere overlain by a IR-transparent, sunlight-absorbing hazy stratosphere. This model [McKay et al., in preparation] reflects $30 \%$ of incident sunlight to space; the hazy stratosphere absorbs a fraction $\beta=1-\exp \left(-\tau_{v}\right)$ of the rest $\left(F_{a}\right)$ and re-emits it (half to space, half to the troposphere) as IR. The IR thermal flux from the surface $\left(\sigma \mathrm{T}_{s}^{4}\right)$ is therefore equal to the sum of the IR flux from the haze $\left(0.5 \beta F_{a}\right)$ and the IR flux from a grey atmosphere heated from below by absorbed sunlight, i.e. (1$\beta) F_{a}\left(1+0.75 \tau_{I R}\right)$.

The model reproduces the present surface temperature for a tropospheric IR optical depth $\tau_{I R}$ of about 4 (roughly the present Rosseland mean opacity) and a haze absorption optical depth $\tau_{v}$ set to 1.1. Fixing an effective emissivity of the haze $\mathrm{e}=0.02$ reproduces the present stratospheric temperature $T_{\text {strat }}=\left(0.5 \beta F_{a} / e \sigma\right)^{0.25}$ of $\sim 170 \mathrm{~K}$.

Figure 1 shows that if $\tau_{v}$ is held fixed at 1.1, Titan warms by about $90 \mathrm{~K}$ for a tenfold increase in luminosity - rather more than predicted by the r-c model. If, however, the haze optical depth is made proportional to temperature (i.e. $\tau_{v}=\mathrm{kT}$, with $\mathrm{T}=\left(\mathrm{T}_{s} \mathrm{~T}_{\text {strat }}\right)^{0.5}$ and $\left.\mathrm{k}=0.0085 \mathrm{~K}^{-1}\right)$ then the dampened response to insolation increase described above can be reproduced, although using this mean temperature $\mathrm{T}$, the r-c model results are in fact best fit by a $\tau_{v}=\mathrm{kT}^{1.4}-$ see figure 1 .

All these temperature rises are probably conservative (cool) in that the IR opacity is held fixed, whereas in reality it may increase with temperature as additional greenhouse gasses are volatilized from the surface (we neglect the uncertain role of clouds).

The $\tau_{v}=\mathrm{kT}^{1.4}$ feedback acts as a significant brake to warming as solar luminosity increases. However, haze production is driven by photochemistry, and hence by the UV flux from the sun. As the sun reddens, (at age $10.9 \mathrm{Gyr}$, the sun's effective temperature drops to 4900K [Sackmann et al., 1993]) the UV flux falls off. With a lower UV flux, the haze production $(\mathrm{k})$ drops which in turn allows more visible radiation to reach the surface ( $\beta$ becomes smaller), indicated schematically in figure 2 , and Titan's surface becomes dramatically warmer.

The only significant effect of the change in the solar spectrum is the drop in UV and hence haze production: we have experimented with the r-c model, and find a $5800 \mathrm{~K}$ black body spectrum gives a temperature profile identical to that for the actual solar spectrum. A $4900 \mathrm{~K}$ black body spectrum,

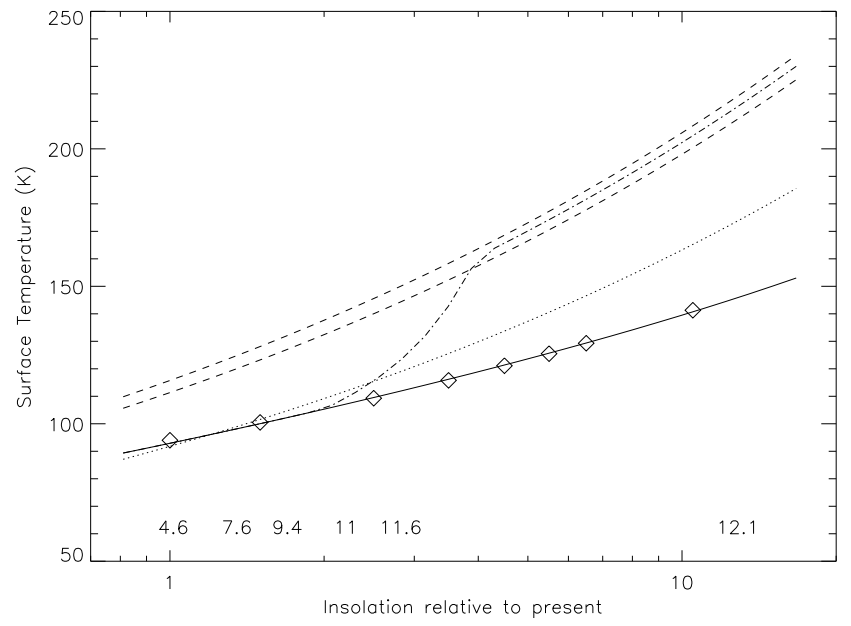

Figure 1. Titan surface temperatures as a function of solar luminosity. The dotted curve shows the results from the simple model described in the text with $\tau_{v}=1.1$; the solid curve has $\tau_{v}=\mathrm{kT}^{1.4}$ and gives a good match to results (diamonds) from the r-c model. The dashed curves have an (arbitrarily) fixed 'red-giant' $\tau_{v}$ of 0.02 (upper) and 0.2 (lower) The probable evolution of Titan's surface temperature is shown by the dash-dot curve, jumping from the $\tau_{v}=\mathrm{kT}^{1.4}$ to 'red giant' curve as the sun's UV flux drops. All the se temperatures are conservative (cool) in that $\tau_{I R}$ is held fixed. The numbers near the bottom are the solar age for various luminosities from Sackmann et al.,[ 1993].

with the same luminosity, yields a slightly (10K) lower stratospheric temperature because this cooler and hence redder spectrum is absorbed less by the small haze particles. More sunlight is now in the near-IR methane absorption bands, however, so the net increase in surface illumination due to a change in spectrum alone is minimal and the temperature only rises by $2 \mathrm{~K}$.

Thus, how warm the surface gets depends essentially only on the haze production. Organic production by UV is two orders of magnitude higher than that by magnetospheric electrons and cosmic rays [Sagan et al., 1984]. Thus a residual haze production of about one hundredth of the present value would be expected with no UV flux at all: this corresponds approximately to the $\tau_{v}=0.02$ curve in figure 1 . If the UV flux scales with the Planck function for $150 \mathrm{~nm}$, then a drop in emission temperature from $5800 \mathrm{~K}$ to $4900 \mathrm{~K}$ gives a factor of 10 drop in haze production, corresponding to the $\tau_{v}=0.2$ curve. In fact, late-type stars are subluminous by a factor of 10 compared to the blackbody flux for their emission temperature [Kasting et al.1997] so a value between 0.2 and 0.02 may be appropriate. In any case, when $\tau_{v}$ is below 1 , the surface temperature is not very sensitive to its exact value, so whether the UV flux drops by a factor of 10 or 100 is unimportant.

\section{Response to Solar Mass Loss}

The sun's diameter and mass change drastically as its spectrum reddens, with Mercury being engulfed [Sackmann et al.,1993] and significant solar mass loss occurring: from 10.91 Gyr age (luminosity $\mathrm{L}=2.21$ times present luminosity $\left.\mathrm{L}_{0}\right)$ to $11.64 \mathrm{Gyr}\left(\mathrm{L}=2.73 \mathrm{~L}_{0}\right)$, the sun loses $0.02 \%$ of its mass; 

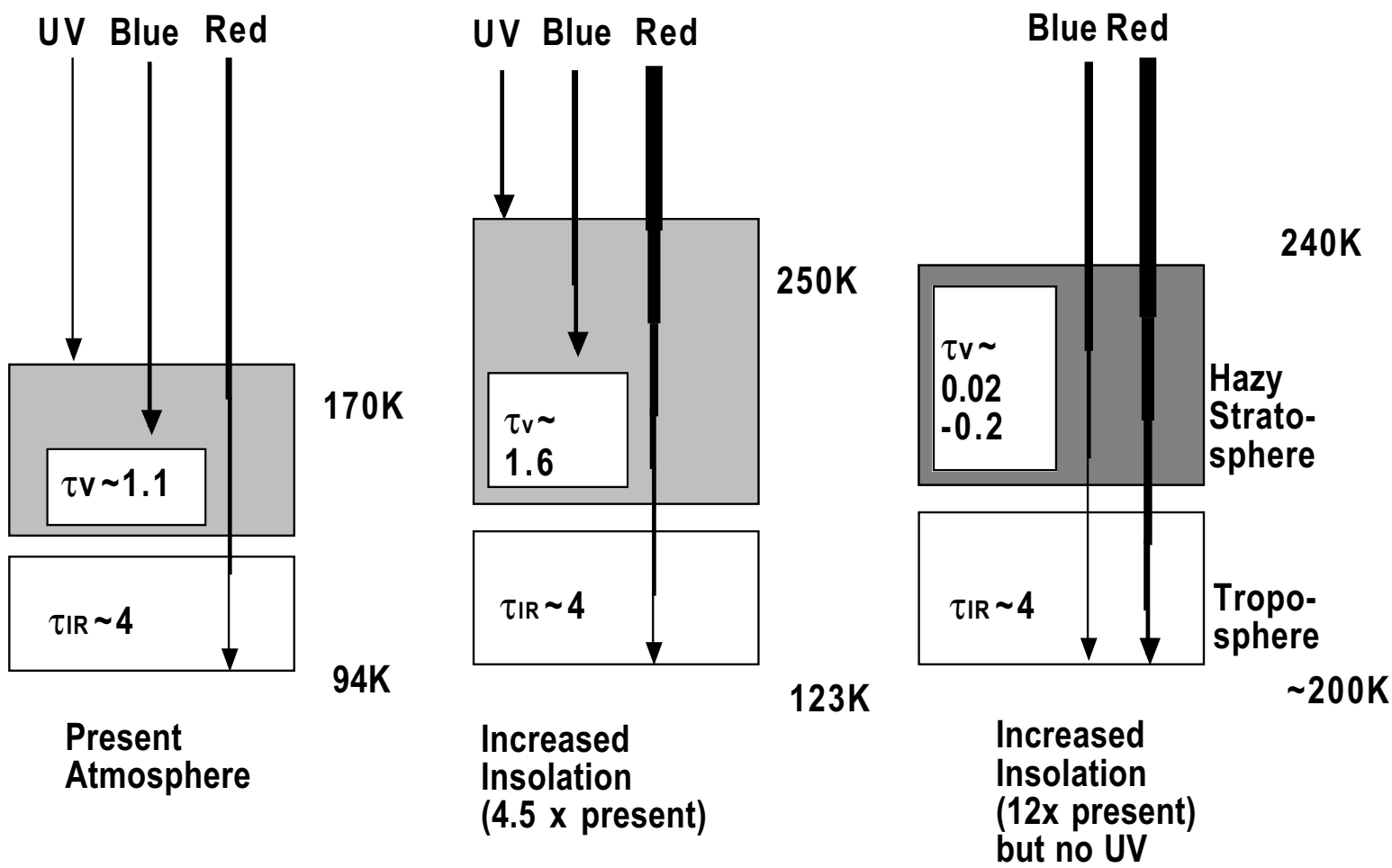

Figure 2. Schematic illustration of the response of Titan's atmosphere to solar evolution. An absorbing haze overlies a greenhouse layer over the surface. Increasing the insolation expands the atmosphere, reducing the fraction of sunlight reaching the lower layer so that the temperature increase is small. Once solar UV dies out, haze production falls and more sunlight penetrates to the surface, although the stratosphere is cooler. Surface and stratospheric temperatures are computed (figure 1) from the simple model described in the text.

from there to $12.088 \mathrm{Gyr}\left(\mathrm{L}=17.3 \mathrm{~L}_{0}\right)$ it loses another $0.37 \%$ and thence to $\sim 12.15$ Gyr it loses another $0.36 \%$. These mass losses correspond to mass fluxes of 16, 350 and $\sim 3000$ times that of the present day solar wind. Loss from Titan's atmosphere [Lammer and Bauer, 1991] due to the solar wind $(1 \mathrm{~g} / \mathrm{s})$ is at present a minor contributor, compared with nonthermal losses due to dissociative ionisation of $\mathrm{N}_{2}$ and dissociative recombination of $\mathrm{N}_{2}^{+}$of $\sim 300 \mathrm{~g} / \mathrm{s}$. Thus, as erosion of Titan's atmosphere scales with solar wind mass flux even the highest rate of solar mass loss given above corresponds to removal of less than $2 \%$ of Titan's atmosphere in 1 Gyr. After 12.15 Gyr age, however, the sun pulsates and loses mass extensively. We have not attempted to model the response of the surface or atmosphere to these (probably catastrophic) effects.

\section{Exobiological Implications}

Depending on the exact IR and haze opacity history, our model results suggest that Titan's surface may easily have $\sim 500$ Myr of aqueous exposure in the far future, much longer than the estimated average of 10-1000 years [Thompson and Sagan, 1992] from impact melting so far in Titan's history. This exceeds the formation time of life on Earth, although due to Arrhenius kinetics (and perhaps the higher viscosity [Kargel et al.1991] of water-ammonia liquids) reactions will proceed more slowly on Titan.

Thus, invoking the presence of ammonia enables the preservation of a liquid aqueous surface under lower insolation levels, due to the antifreeze effect of the ammonia and the greenhouse warming of the atmosphere. To discriminate this scenario from a 'primordial soup' under a $\mathrm{CO}_{2}$ greenhouse, we are tempted to call this colder, more reducing and alkaline variant a 'primordial gazpacho'.

Evolved stars with low UV fluxes could sustain a second 'habitable' zone, outside the conventional one, in which preand proto-biotic chemistry may proceed.

\section{Conclusions}

Temperatures in the outer solar system can be sustained above the water-ammonia melting point by a methane greenhouse effect. Lower insolation levels in turn allow the ammonia and methane to last longer against photolysis and escape than they would under terrestrial UV flux and temperature conditions. A significant antigreenhouse cooling effect due to haze produced by methane photolysis is augmented at higher fluxes, due to expansion of the atmosphere and increase of the aerosol column depth. This feedback, however, is largely eliminated in the sun's red giant phase. Although in our own solar system Titan will not have such an episode for some 6 Gyr, other nearby stars are approaching the end of their main sequence lifetime (e.g. 51 Pegasi). It might be that, before life on Earth is extinguished by a rising sun [Caldeira and Kasting, 1992], steps towards life could begin on icy worlds surrounding neighbouring stars.

Acknowledgments. RDL acknowledges support from the Cassini project. JIL and CPM are supported by the NASA Geology/Geophysics and Planetary Atmospheres Programs. We thank 
the referees for useful comments and Darren Williams for encouragement.

\section{References}

Caldeira K. and Kasting, J. F., The Life Span of the Biosphere Revisited, Nature 360 721-723 (1992).

Croft, S. K., Lunine, J. I. and Kargel, J. S., Equation of State of ammonia-water liquid: derivation and planetological applications Icarus 73 279-293 (1988).

Haudenschild, A., Environmental Requirements, JPL Space Programs Summary 37-64 3 (1970).

Lammer, H. and Bauer, S. J., Nonthermal Escape from Mars and Titan, J. Geophys. Res. 96 1819-1825 (1991).

Kargel, J. S., Croft, S. K., Lunine, J. I. and Lewis, J. S., Rheological Properties of Water-Ammonia Slurries, Icarus 89 93-112 (1991).

Kasting, J. F., Whittet, D. C. B. and Sheldon, W. R., Ultraviolet Radiation from $\mathrm{F}$ and $\mathrm{K}$ Stars and Implications for Planetary Habitability, Origins of Life and Evolution of the Biosphere $\mathbf{2 3}$ 413-420 (1997).

Kiehl, J. T. and Dickinson, R. E., A Study of the Radiative Effects of Enhanced Atmospheric $\mathrm{CO}_{2}$ and $\mathrm{CH}_{4}$ on Early Earth Surface Temperatures, J. Geophys. Res 92 No. D3 2991-2998 (1987).

Kuhn, W. R. and Atreya, S. K., Ammonia Photolysis and the Greenhouse Effect in the Primordial Atmosphere of the Earth, Icarus 37 207-213 (1979).

McKay, C. P., Pollack, J. B. and Courtin, R., The Greenhouse and Antigreenhouse Effects on Titan, Science 253 1118-1121 (1991).

McKay, C. P., Pollack, J. B. and Courtin R., The Thermal Structure of Titan's Atmosphere, Icarus 80 23-53 (1989).

McKay, C. P., Lunine, J. I. and Courtin, R., Coupled AtmosphereOcean Models of Titan's Past, Icarus 10288 (1993).
Sackmann, I-J., Boothroyd, A. I. and Kraemer, K. E., Our Sun. III Present and Future, Ap. J. 418 457-468 (1993).

Sagan, C. and Mullen, G., Earth and Mars: Evolution of Atmospheres and Surface Temperatures, Science 177 52-54 (1972).

Sagan, C., Khare, B. N. and Lewis, J. S. Organic Matter in the Saturn System. in Saturn (eds. T. Gehrels and M. S. Matthews) University of Arizona Press, 788-810 (1984).

Sagan, C. and Chyba C., The Early Faint Sun Paradox: Organic Shielding of Ultraviolet-Labile Greenhouse Gases Science $\mathbf{2 7 6}$ 1217-1221 (1997).

Samuelson, R. E., A Radiative Equilibrium Model of Titan's Atmosphere, Icarus 53 364-387 (1983).

Shapiro, R. and Feinberg, G. Possible forms of life in envrionments very different from the Earth. in Extraterrestrials: Where are they? (ed. B. Zuckerman and M. H. Hart). Cambridge University Press, pp. 165-172 (1995).

Thompson W. R. and Sagan, C., Organic Chemistry on Titan - Surface Interactions, Symposium on Titan European Space Agency ESA SP-338, 167 (1992).

Williams, D. M., Kasting, J. F. and Wade, R. A., Habitable Moons around Extrasolar Giant Planets, Nature 385 234-236 (1997).

R. D. Lorenz and J. I. Lunine, Department of Planetary Sciences, Lunar and Planetary Laboratory, University of Arizona, Tucson, AZ 85721. USA. (email rlorenz@lpl.arizona.edu, jlunine@lpl.arizona.edu)

C. P. McKay, NASA Ames Research Center, Moffett Field, CA 94035, USA. (email: mckay@gal.arc.nasa.gov)

(Received May 13, 1997; revised August 9, 1997; accepted October 7, 1997.) 